\title{
Specialized microbial metabolites: functions and origins
}

\author{
Julian Davies \\ The period from 1950 to date brought about a revolution in the pharmaceutical industry: the use of large-scale fermentation \\ methods to produce drugs (mostly antibiotics). The industrial modification of natural biosynthetic processes led to production \\ methods capable of generating massive amounts of therapeutic agents. Natural products became industrial products. This \\ transition took place in complete absence of any knowledge of the roles and functions of small molecules in nature. This \\ discourse attempts to redirect enquiry into the natural roles of these molecules and the ways in which they regulate microbial \\ populations.
}

The Journal of Antibiotics (2013) 66, 361-364; doi:10.1038/ja.2013.61; published online 12 June 2013

Keywords: antibiotic; biosynthesis; cyclic dipeptides; origins; Parvome; signaling

\section{INTRODUCTION}

There is a superabundance of bioactive small molecules produced by all living species in the Biosphere: the 'Parvome.' What are the environmental roles of this extraordinarily rich collection of bioactive organic compounds? For more than 50 years they have been of interest solely in their roles as antibiotics, and although a miraculous discovery, this was an anthropocentric concept that has restricted the proper study of the natural functions of these molecules. As noted by Stuart Schreiber, the natural reservoir of bioactive low-molecular-weight compounds should be considered as an integral component of the central dogma of biology. ${ }^{2}$ There are three processes in this principle: physical interactions between nucleic acid bases in replication and transcription, reading combinations of the four letters of the genetic code for protein production, and finally molecule-based ligand/receptor interactions mediated by specialized metabolites and macromolecules. Some microorganisms devote up to $15 \%$ of their genome content to the production of specialized microbial metabolites (SMs).

This short essay discusses specialized metabolites and their involvement in numerous aspects of cell biology, together with speculation on the evolution of this type of metabolism. My article is written as a tribute to the late Leo Vining, a fine gentleman and a leading exponent in the field of streptomycete biology, with whom I had an intense breakfast discussion on the topic of 'metabolism' at a CIBA Foundation Symposium held in London in 1992. ${ }^{3}$

\section{A BRIEF HISTORY OF ANTIBIOTICS}

Historically, the study of naturally-occurring bioactive organic molecules was driven mainly by chemical curiosity, but recently and more intensively by the search for therapeutics produced in nature. These compounds have provided significant structural and synthetic challenges to chemists, and many valuable medicinal products have been identified and characterized. For example, plant alkaloids such as quinine and simple compounds such as aspirin (the first non steroidal anti-inflammatory drug) were miraculous therapeutic findings in the 1800s. The discovery of antibiotics in the mid-1940s by Fleming and colleagues (penicillin), and later by Schatz and Waksman (streptomycin) opened the floodgates to natural product discovery. These drugs provided the first effective treatments for terrifying microbial diseases that were responsible for the major plagues of human history. Coincidentally, the discovery of antibiotics birthed the modern pharmaceutical industry. The identification and characterization of the major microbial pathogens with the concurrent discoveries of 'miracle' drugs from microbes led to an extraordinary period of discovery: the antibiotic era. Between 1945 and the present day, it is probable that hundreds of thousands of SMs have been isolated and tested for their ability to treat bacterial, fungal, parasitic and viral infections of humans and domestic animals. It is worth noting that clinical trials of antibiotics were somewhat perfunctory in the 1950s; they were introduced into clinical practice shortly after their purification and demonstration of nonobvious toxicity in animals.

The suppression of microbial infections by these products has played a significant role in increasing human life expectancy. It should be recognized that antimicrobials were not the only factors in this revolution: improvements in public health, sanitation and supplies of clean water accompanied the antibiotic era. Applications to human and animal health have dominated the field of natural product research.

During the exciting and exponential phase of antibiotic (SM) discovery, very little was done in the way of research on the roles of 
this chemical cornucopia in the natural microbial world. The microorganisms producing such molecular wealth were denizens of a wide range of soils and other environments. Compounds were screened for antibiotic activity in the laboratory with the assumption that they were involved in intermicrobial competition for nutrients or space in soil ecosystems. Anthropocentricity guided natural product studies, and the fact that there had been no contact with common human or animal microbial pathogens was of no consequence Nonetheless, this concept provided the raison d'etre for antibiotic discovery, transformed medicine by providing potent therapeutics and saved millions of lives that were previously threatened by untreatable infections. All this, with minimal improvement in the understanding of environmental microbiology.

Investigation of the natural roles of SMs is not easy to perform, and is also limited in scope. This is changing with the introduction of metagenomic analyses of a variety of environments. An important recent stimulus for research on the natural activities of SMs has come from the identification, some 30 years ago, of small-molecule intercellular signaling processes in microbes. Studies by a number of microbiologists in the discovery and characterization of quorum sensing (QS) molecules and related systems have changed popular notions of the roles of low-molecular-weight compounds, their production and their roles in bacterial communities. ${ }^{4}$ Various QS agents have been identified from a wide range of bacterial genera (also in yeast and fungi). Interestingly, the process is common in antibioticproducing organisms: in 1967 Khokhlov and coworkers identified A-factor, a substituted $\gamma$-butyrolactone made by Streptomyces griseus. ${ }^{5}$ This was the first of a large family of signaling SMs to be identified in streptomycetes. A-factor was subsequently shown to bind to a cytoplasmic receptor and to regulate key metabolic processes in the target organism. These included morphological changes (spore formation), the production of pigments and other SMs.

Small molecule signaling and other forms of cell-cell interaction, both agonistic and antagonistic, are characteristic of all forms of microbial life, and are responsible for the majority of interactions within and between microbiomes. Multicellularity is a bacterial lifeform. Many low-molecular-weight compounds participate in interand intra-species and even cross-kingdom interactions, such as those mediated by the adrenergic hormones (such as epinephrine), plant hormones (such as auxin) and microbial products (such as homoserine lactones and $\beta$-lactams). These functional network interactions between cells and organisms in nature, including eukaryotic hosts, are complex.

\section{PARACELSUS: 'THE DOSE MAKES THE POISON'}

It has been shown that SMs (antibiotics) have dual roles and display unsuspected activities at low concentrations: analyses of these phenomena have led to questions about the biological roles of SMs. Are they naturally antibiotics? Sub-MIC activities can be monitored by the concentration-specific patterns of transcription induced by a wide variety of molecules with antibiotic activity. Specific differences in terms of patterns and levels of transcription were found to depend on the concentration and nature of the molecular target (receptor). Similarities were noted between compounds affecting specific cellular targets (for example, ribosomes, DNA gyrases, RNA polymerase, etc.) and the SM inducer. ${ }^{6}$ Increasing concentrations lead to complete blockade, as predicted by Paracelsus. Studies of the therapeutic targets of the molecules examined in response to subinhibitory concentrations have provided an increased understanding of targetreceptor interactions and their natural roles: the use of resistant mutants supplies more detailed information on SM/target responses.
As a result most of the compounds identified as antimicrobials probably have roles in natural cell physiology and intercell signaling. They may also maintain microbial population structures (microbiomes) in health and disease. Detailed examination of the effects of antibacterial agents on mammalian, plant, and other eukaryotic cells can be anticipated to lead to findings of therapeutic use.

As an example, the effects of the bacterial protein synthesis inhibitor erythromycin on TLR4 responses is modulated by a single amino-acid change in a ribosomal protein. ${ }^{7}$ Surprisingly, this antibiotic also binds to the human motilin receptor, and this interaction is responsible for the intestinal disturbances occurring during treatment with erythromycin. Similar dual prokaryoteeukaryotic effects have been demonstrated with rifampicin and other therapeutic agents. An especially interesting and clinically-validated example is that of rapamycin, a compound isolated in an antifungal screen but discovered to be an extraordinarily potent immunosuppressive agent. The use of rapamycin and derivatives has revolutionized the practice of organ transplantation in humans. Identification of novel cross-species interactions of SMs will likely be a topic of continuing clinical importance; it will also contribute to a better understanding of mixed biological systems and their interactions.

Although the Parvome is enormously diverse, the available knowledge of its molecular content is strongly biased on microbial SMs. The reason is obvious, as drug discovery and development was driven by a search for compounds produced by bacteria and fungi. A treasure trove of bioactive SMs is now available. Half a century of discovery effort by the pharmaceutical industry has revealed but a tiny fraction of the content of the Parvome. Despite the fact that the SM catalogue is far from complete, the products obtained have revolutionized the therapy of microbial and other diseases. Other applications come from studies of the toxic effects of SMs that have provided a range of drugs for the treatment of cancer and other chronic ailments. Daunorubicin is a good example of a successful anticancer drug, and lovostatin a much-used cholesterol-lowering agent. Many benefits have evolved from more than 50 years of SM discovery, overproduction, clinical testing and trials for new antibiotics, but investigations of the natural roles of SMs were largely ignored. Academic studies were based on the assumption of roles of these active compounds in fictional intermicrobial warfare, in tune with industry. The sine qua non was that compounds studied must demonstrate activity as antibiotics in the laboratory.

\section{THE FLOOD: BIOSYNTHESIS AND FUNCTION}

Before the genomic era, studies of SM biosynthesis were limited and difficult, mostly involving random mutation, purification of pathway enzymes and reconstruction of biosynthetic steps in the laboratory. Modern high-throughput DNA sequencing has exposed the vast 'hidden' biosynthetic content of microbial and other genomes: the SM reservoir is immense. ${ }^{8}$ A turning point came with the heroic completion of the sequence of the Streptomyces coelicolor genome: this revealed the presence of many potential biosynthetic gene clusters encoding previously unsuspected compounds. ${ }^{9}$

Triggered by this exciting revelation, others followed and DNA sequencing and associated analytical approaches revealed many novel biosynthetic pathways and previously unknown SMs in a variety of bacteria and fungi, and also in environmental microbiomes. Complete genomes of many antibiotic-producing bacteria have been determined: this applies to the Actinobacteria, the Gram-negative Myxococci and others. The complete genome sequence of the important environmental organism, Rhodococcus jostii RHA1, which was not known to produce SMs, revealed the biosynthetic potential to 
produce more than a dozen nonribosomal peptides (NRPs) and polyketide (PK) molecules. The list includes multiple novel pathways identified in complex fungi such as the Aspergilli. ${ }^{10}$ Even bacterial pathogens make SMs!

Identification of the complex biosynthetic routes for novel SMs by genome sequence analysis has also provided clues on the regulatory processes involved in the synthesis of structurally diverse bioactive molecules. The list goes far beyond NRP and PK clusters to include previously unrecognized (cryptic) biosynthetic pathways. Improvements in mass spectroscopy, NMR, combined with high-resolution separation methods have enhanced the process of structure determination. The biosynthetic pathways may involve 20 or more discrete enzymatic steps from simple precursors. They are tightly regulated and involve high-molecular-weight, multifunctional enzymes that act processively to generate diverse chemistry. The core structures produced are frequently decorated by reactions such as glycosylation, alkylation or other modifications. The two most studied classes of SMs are the NRPs and PKs, some of these giant gene clusters come close to the size of a small bacterial genome! These two groups of molecules are probably universal; NRPs and PKs (and their hybrids) are found in the intestinal tract, probably the products of hostassociated microbiomes. They are likely involved in host-microbe interactions in the gut. Toxins made by bacterial pathogens (such as Escherichia coli, Shigella sp. and Salmonella sp.) are often plasmidencoded NRPs or PKs. The almost-universal distribution of these complex pathways is convincing proof of the functional importance of SMs in the biology of all living organisms. Identifying their roles will have an enormous impact on human and animal health and diseases in the future.

\section{WHY SPECIALIZED?}

There has been much discussion on the definition of secondary metabolism and secondary metabolites, and it is time to end this! The definition is based on years of anthropocentric interpretation of microbial physiology in terms that describe microbial behavior as an analog of human behavior. Under laboratory conditions, SMs were considered to be produced only during late phases of growth, and were thus described as secondary metabolites to distinguish them from growth-related primary metabolism. The SMs were considered to provide functions ancillary to the metabolic events associated with nutrient metabolism, cell division, propagation and the like. These identifications were carried out under laboratory conditions that rarely came close to representing nature.

Microbes in natural environments have slow growth rates and exist primarily in stationary phase; they are rarely pure cultures. Metagenomic analyses of soils, sediments and the like have shown that they consist of complex, mixed communities (microbiomes) that include hundreds of environmentally defined, discrete genera growing in close contact. Since the time of Waksman (who coined the word antibiotic), SMs were the artillery in hostile interactions within and between microbes and other organisms. With an increasing understanding of the genetic and biochemical investment in the production of SMs, it is considered appropriate to refer to these molecules as specialized metabolites to emphasize their importance in microbial ecology. The specific roles of these molecules are not known at this time. But in no sense are they secondary! ${ }^{11}$

Attempts to detect antibiotic-like compounds in soils, etc., have so far been unsuccessful. Are they not made, not secreted, present at undetectably low concentrations, bound tightly to cellular or soil particulate material? How is their production regulated? And what are the natural triggers of this process? In situ meta-transcriptomic and meta-proteomic studies should shed answer to some of these questions. It is known that for the manufacture of unnaturally high yields of SMs required for therapeutic development, producing strains are exposed to many cycles of mutation and the resulting 'improved' industrial strains are then subjected to large-scale, unnatural growth in very rich media to obtain the quantities required for commercial production. Although the ultimate in anthropocentricity, this approach has successfully provided the clinical-grade material needed to support worldwide use. The survival of such genetically modified organisms in the wild has not been studied.

\section{SMALL-MOLECULE ORIGINS: WHICH CAME FIRST, THE CHICKEN (TARGET) OR THE EGG (SM)?}

The presence of bacteria on this planet has been dated back to some 3.5 billion years. The evolution of the biosynthetic pathways for SMs is a mystery. They are certainly ancient and estimates suggest that compounds such as the PK erythromycin may be a billion years old. ${ }^{12}$ Precursors of SMs, such as amino acids, are older and thought to have been made by primordial reactions or delivered to Earth by meteorites (carbonaceous materials) from space. Given the importance of amino acids in structures involved in cell shape and function, and their presence as components of many SMs, the NRPs are probably the oldest SMs. Molecular classes like the PKs and aminoglycosides are also deemed to be ancient, although probably not of NRP antiquity.

Several different schemes have been proposed for the prebiotic formation of the essential biological monomers. Following the predictions of Oparin, Haldane and others, the laboratory prebiotic atmosphere studies of Stanley Miller and Harold Urey provided convincing evidence that protein and nonprotein amino acids could have been formed in the earth's prebiotic atmosphere. As confirmation of this, many prebiotic reaction products have been identified among the organic constituents of carbonaceous meteorites. Interestingly, reanalysis of Miller's reactions using higher-resolution detection procedures has revealed the presence of additional protein amino acids and also amino acids present in nonribosomal peptides. For example, the rare nonprotein amino acids 3-methylglutamic acid and L-kynurenine are found in NRPs such as daptomycin. These findings lend credence to the notion that the building blocks for complex organic molecules were available, and that NRPs could have been produced.

When pondering the origins of bioactive low-molecular-weight compounds, it is useful to keep in mind their modern synthetic and regulatory roles in bacteria. Can one extrapolate back to reveal the potential roles in bacterial evolution? Many SMs target the 'central dogma' processes in cells: replication, transcription and translation. Their activities are concentration-dependent and at low doses these molecules may enhance macromolecular reactions. Antibiotics that interfere with translation have been observed to stimulate bacterial growth at low concentrations. Some translation inhibitors stimulate peptide bond synthesis in vitro under nonideal conditions, for example, low amino-acid concentrations.

Assuming that SMs or their precursors facilitated prebiotic macromolecular processes during the early stages of biological evolution: these 'factors' would have been replaced as the efficiency of macromolecular syntheses improved. Combinations of peptides with small RNA molecules might have provided catalytic frameworks that facilitated the (inefficient) formation of peptide bonds. ${ }^{13}$ This may have been the original 'role' of SMs identified as antibiotics. Combinations of SMs with diverse RNA molecules may have generated RNP 'complexes' that enhanced or accelerated the formation of peptide bonds. As such complexes evolved increased 
efficiency, the SMs could be functionally displaced, but the binding sites survived on the proto-ribosome, to become sites for inhibition. Other forms of peptide and SM complexes could have provided catalytic core structures for the synthesis of other macromolecules, such as the RNA.

Speculations about chemical evolution are easy to propose, hard to confirm and difficult to overturn!

\section{CYCLIC DIPEPTIDES: NUISANCE OR IMPORTANCE?}

There exists a huge population of simple amino-acid derivatives, the cyclic dipeptides (CDP) found in many living organisms; microbes, plants and animals. There are several excellent reviews that describe the world of CDPs. ${ }^{14}$ They are produced by two major biosynthetic routes: as products of NRP synthetases, or more interestingly, by a unique enzymic process employing cyclopeptide synthases (CDPS) that use charged aminoacyl-tRNAs as a substrate. The latter forms an interesting link between aa-tRNA-synthetase-based protein synthesis and other processes for peptide bond formation. Genome mining indicates the presence of CDPS genes in more than 50 different bacteria, also fungi and protozoa.

CDPs have been detected in primordial soup reactions and carbonaceous meteorites, and are probably the most ancient peptides: they may contain nonprotein amino acids. The CDPs provide structural cores for a number of complex natural products (diketopiperazines) that include the aranotins, bicyclomycin, chaetocin, cyclomarazines, gliotoxin and verticillin $\mathrm{B}$, to name a few. Specific tailoring enzymes are employed for these decorations.

As primordial peptides, the CDPs could have had many roles in the evolution of cell structure and metabolism, and also as catalysts. A number of CDPs have been reported to enhance bacterial functions such as growth, differentiation, and cell-cell signaling, but by and large they are compounds seeking defined role(s). In some instances CDPs act in synergy with each other. There is some evidence that they are activators or inhibitors of QS reactions, but this is controversial. Are they simply evolutionary relics or do CDPs have defined modern functions? They have been shown to be associated with bacterial virulence: how did this process evolve? Given their antiquity, might they have had catalytic roles in cell-cell interactions?

Natural product chemists consider CDPs to be nuisance compounds, as they are ubiquitous and present no structural challenge. However, in recent years interest in CDP biology has increased because of their novel mechanism of biosynthesis. It is rare to find an organism producing a single class of CDP, and some bacteria have been shown to produce more than six different variations of these molecules.

CDPs have been claimed to have a wide range of biological activities in the laboratory: antitumor, antiviral, antibacterial, antifungal, immunosuppressive agents, neurological, as signaling molecules, as well as endocrine effects, although identification of their natural roles has not been confirmed. They have also been proposed to have a role in bacterial pathogenesis; ${ }^{15}$ if confirmed, might this imply that DKPs are precursors of bacterial virulence factors?

\section{CONCLUSIONS OR DELUSIONS?}

Bioactive small molecules determine the physiological characteristics of Prokaryotes, Archaea and Eukaryotes and have important roles in the intra- and extra-cellular interactions that exist within and between cells and organisms.

The Biosphere is populated by an enormous number and variety of bioactive small molecules. The origins, functions and applications of this chemical treasure trove have been largely ignored, apart from those few compounds commercialized for their therapeutic activities, and there have been many! In addition to their success in the control of common infectious diseases and the provision of novel treatments for chronic ailments, SMs have changed human history and biology! They are truly evolutionary molecules.

Nonetheless, little is known of the origins of bioactive SMs and their (many?) roles in the development and maintenance of complex biological systems.

Finally, despite the astonishing success of SMs in generating improvements in human and animal health over the past half-century, there is a major downside. Their use has been responsible for the global-wide pollution of the surface of Earth with toxic molecules and their production wastes. This has created an insidious evolutionary development: that of widespread antibiotic resistance in the environment. The past half-century has seen the industrial production and worldwide distribution of millions of tons of bioactive small molecules, most of them microbial products, but also significant amounts by chemical synthesis that have combined to irrigate the surface of the biosphere with constant chemical contamination. They are one of the principal man-made contaminants in the environment, and in addition to providing constant selection for antibiotic resistant, organisms have other undesirable environmental effects. They have impacted natural microbial ecology in ways and extents that cannot be estimated.

1 Davies, J. \& Ryan, K. S. Introducing the parvome: bioactive compounds in the microbial world. ACS Chem. Biol. 7, 252-259 (2012).

2 Schreiber, S. L. Small molecules: the missing link in the central dogma. Nat. Chem. Biol. 1, 64-66 (2005)

3 Secondary Metabolites: their Function and Evolution (eds Chadwick, D. J. \& Whelan, J.) (Ciba Foundation Symposium; Wiley, Chichester, 1992).

4 Bassler, B. L. How bacteria talk to each other: regulation of gene expression by quorum sensing. Curr. Opin. Microbiol. 2, 582-587 (1999).

5 Khokhlov, K. S. et al. The A-factor, responsible for streptomycin biosynthesis by mutant strains of Actinomyces streptomycini. Dokl. Akad. Nauk. SSSR. 177, 232-235 (1967)

6 Yim, G., Wang, H. H. \& Davies, J. Antibiotics as signaling molecules. Phil. Trans. $R$ Soc. B. 362, 1195-1200 (2007).

7 Oldenburg, M. et al. TLR13 recognizes bacterial 23S rRNA devoid of erythromycin resistance-forming modification. Science 337, 1111-1115 (2012).

8 Walsh, C. T. \& Fischbach, M. A. Natural products version 2.0: connecting genes to molecules. J. Am. Chem. Soc. 132, 2469-2493 (2010).

9 Bentley, S. D. et al. Complete genome sequence of the model actinomycete Streptomyces coelicolor A3(2). Nature 417, 141-147 (2002).

10 Sanchez, J. F., Somoza, A. D., Keller, N. P. \& Wang, C. C. Advances in Aspergillus secondary metabolite research in the post-genomic era. Nat. Prod. Rep. 29, 351-371 (2012).

11 Price-Whelan, A., Dietrich, L. E. \& Newman, D. K. Rethinking 'secondary' metabolism: physiological roles for phenazine antibiotics. Nat. Chem. Biol. 2, 71-78 (2006).

12 Baltz, R. H. Genomics and the ancient origins of the daptomycin biosynthetic gene cluster. J. Antibiot. (Tokyo) 63, 506-511 (2010).

13 Davies, J. What are antibiotics? Archaic functions for modern activities. Mol. Microbiol. 41, 227-1232 (1990).

14 Belin, P. et al. The nonribosomal synthesis of diketopiperazines in tRNA-dependent cyclodipeptide synthase pathways. Nat. Prod. Rep. 29, 961-979 (2012).

$15 \mathrm{Li}$, J. et al. Lactobacillus reuteri-produced cyclic dipeptides quench agr-mediated expression of toxic shock syndrome toxin-1 in staphylococci. Proc. Natl Acad. Sci. USA 108, 3360-3365 (2011). 